Projets

de paysage

\section{Projets de paysage}

Revue scientifique sur la conception et l'aménagement de l'espace

$18 \mid 2018$

Paysage et didactique

\title{
Le paysage pour aborder les interactions entre environnement alpin et sociétés
}

Retour sur une expérience pédagogique menée à l'université de Genève (Suisse)

The landscape as a means of addressing interactions between the alpine environment and society - Feedback on a teaching experiment conducted at the University of Geneva (Switzerland)

Sylvie Paradis, Stéphanie Girardclos, Cédric Lambert, Mathieu Petite et Marjorie Perroud

\section{OpenEdition}

\section{Journals}

Édition électronique

URL : http://journals.openedition.org/paysage/1144

DOI : 10.4000/paysage. 1144

ISSN : 1969-6124

\section{Éditeur :}

École nationale supérieure du paysage de Versailles-Marseille, Institut national des sciences appliquées Centre Val de Loire - École de la nature et du paysage, École nationale supérieure d'architecture et de paysage de Bordeaux, École nationale supérieure d'architecture et de paysage de Lille, Agrocampus Angers

Référence électronique

Sylvie Paradis, Stéphanie Girardclos, Cédric Lambert, Mathieu Petite et Marjorie Perroud, « Le paysage pour aborder les interactions entre environnement alpin et sociétés », Projets de paysage [En ligne], 18| 2018, mis en ligne le 11 juillet 2018, consulté le 28 novembre 2019. URL : http:// journals.openedition.org/paysage/1144; DOI : 10.4000/paysage.1144

Ce document a été généré automatiquement le 28 novembre 2019.

Projets de paysage 


\section{Le paysage pour aborder les interactions entre environnement alpin et sociétés}

Retour sur une expérience pédagogique menée à l'université de Genève (Suisse)

The landscape as a means of addressing interactions between the alpine environment and society - Feedback on a teaching experiment conducted at the University of Geneva (Switzerland)

Sylvie Paradis, Stéphanie Girardclos, Cédric Lambert, Mathieu Petite et Marjorie Perroud

1 Les auteurs ont mené un exercice réflexif, au cours de l'année 2017 entre collègues de différentes disciplines et de plusieurs facultés, intervenant dans la Maîtrise universitaire en sciences de l'environnement (MUSE). Ce master de l'université de Genève, créé en 2007, est axé sur l'apprentissage des enjeux environnementaux dans leurs différentes dimensions sociales, naturelles et économiques. Lors du premier semestre, un module d'enseignement intitulé « Interdisciplinarité et immersion » initie le programme du MUSE, au sein duquel deux enseignements introduisent ensemble des concepts et méthodes en utilisant le cas pratique de l'environnement alpin. Il s'agit du cours $\left(4 \mathrm{ECTS}^{1}\right)$ et de l'atelier (8 ECTS) «Environnement alpin et sociétés » (ISE, 2017), créés à l'occasion d'une réforme du MUSE (Efrancey Dao et Wildi, 2013) sur la base d'enseignements auparavant dispersés dans un tronc commun. Le cours et l'atelier comportent plusieurs modules disciplinaires, majoritairement en sciences naturelles (figure 1). La structuration de ces deux enseignements, dispensés dès l'automne 2015, a mené à la création d'un nouveau module intitulé "Enjeux paysagers », objet de notre réflexion. 
Figure 1. Disciplines et modules enseignés dans le cours et l'atelier « Environnement alpin et sociétés » du MUSE

Image 1000000000000A2F0000049BED940B1A43368B08.jpg

2 Sans remettre en cause la pertinence de ce nouveau module, quelques enseignants volontaires se sont interrogés a posteriori sur l'interdisciplinarité du cours et de l'atelier "Environnement alpin et sociétés », et plus spécifiquement sur la capacité de la notion du paysage à stimuler des interactions plus importantes entre les disciplines, autant au niveau des dimensions cognitives (savoirs et apprentissages) que pragmatiques (méthodes et mise en œuvre de l'interdisciplinarité) (Rege Collet, 2003).

3 Pour mener cette analyse, les auteurs, d'une part, ont procédé à une enquête par questionnaire réalisée au début de l'année 2017 auprès d'étudiants et d'enseignants du MUSE des volées 2015 et 2016 et, d'autre part, se sont appuyés sur les évaluations annuelles des cours menées par l'université de Genève (ADEVEN², 2015 et 2016). Les éléments d'analyse liés à une réforme du programme ont également été étudiés (Efrancey Dao et Wildi, 2013). L'exercice réflexif a été complété par des discussions et des réunions de travail durant l'année 2017. En sus de l'analyse portant sur les améliorations pédagogiques, les auteurs évaluent en quoi cette expérience itérative d'ouverture à la thématique du paysage pourrait améliorer l'interdisciplinarité, voire une transdisciplinarité, entre les champs enseignés.

\section{Un contexte pédagogique spécifique}

4 Le MUSE est une formation interdisciplinaire qui est proposée par l'Institut des sciences de l'environnement (ISE), conjointement avec la faculté des sciences, la faculté des sciences de la société et la faculté d'économie et de management de l'université de Genève. Divers domaines de l'actualité environnementale y sont étudiés, tels que le climat, l'énergie, l'eau, la biodiversité, la gouvernance des ressources ou le développement durable ${ }^{3}$. Le MUSE propose une approche "globale», au sens systémique et holistique du terme. Ce master est ouvert à un public d'étudiants issus des sciences exactes et naturelles comme des sciences humaines, économiques et sociales, qui décident de poursuivre leur cursus académique en sciences de l'environnement. La diversité des parcours est amplifiée par une proportion significative d'étudiants ayant effectué leur formation de bachelor à l'étranger, et dont les cultures sont très variées. Dès le début de la formation, l'enjeu est donc de veiller à ce que les candidats comprennent des notions et méthodes (de base) des disciplines mobilisées, malgré leurs origines diverses, et ainsi de favoriser un esprit d'ouverture vers l'ensemble des disciplines enseignées.

Dans cet esprit, le cours et l'atelier «Environnement alpin et sociétés » explorent, à travers un panel de concepts et de méthodes complémentaires, l'environnement et ses interactions avec les sociétés vivant dans les Alpes suisses. Le cours amorce les différents modules disciplinaires, comme la glaciologie ou l'écologie humaine, et pose les bases, méthodes et concepts nécessaires qui vont progressivement être déployés dans l'atelier, lieu privilégié de l'application et de l'expérimentation sur le terrain. Ici, il ne s'agit pas de couvrir tous les domaines, mais seulement d'esquisser une diversité nécessaire à l'expérimentation. Cet atelier est mis en place sous forme d'une semaine 
intensive d'acquisitions de données, d'enquêtes et d'analyses, où les étudiants côtoient les enseignants au quotidien et travaillent en groupes de 3-4 personnes ${ }^{4}$.

6 L'interdisciplinarité est envisagée ici comme le croisement fécond des regards disciplinaires, au sens d'une mise en relation des différentes disciplines (Reverdy, 2015) en particulier lors de l'exercice pratique. Elle a lieu par comparaison et est donc consécutive des enseignements et modules d'atelier disciplinaires, au sens où l'on instaure une certaine culture qui s'emploie à enrichir les disciplines entre elles et à mettre à l'épreuve les savoirs disciplinaires passant par une formalisation (Resweber, 2011) lors de la rédaction du rapport par les étudiants.

Durant l'atelier, pour parvenir à pallier l'hétérogénéité des savoirs chez les étudiants et promouvoir un échange interdisciplinaire, la méthode du Jigsaw teaching (Aronson, 1978 et 2002; Clarke, 1994) est appliquée. En effet, cette méthode promeut les interactions entre les étudiants et les enseignants, mais aussi entre les étudiants euxmêmes (Saint Fleur et al., 2016). Le Jigsaw teaching permet une transmission entre étudiants de savoirs et de méthodes au travers d'« experts " (au sens de responsables disciplinaires et relais). Chaque étudiant sera ainsi désigné comme "expert » d'une discipline auprès des autres étudiants de son groupe, répartition qui se fait sur la base de leur formation disciplinaire. Les « experts " reçoivent une formation d'une demijournée des enseignants de la discipline désignée, ils sont ensuite responsables de la transmission de ces connaissances et instructions de travail aux autres étudiants de leur groupe.

Cette modalité pédagogique a été choisie pour favoriser les échanges entre étudiants d'un même groupe, chacun ayant une responsabilité envers les autres, et pour éviter que chacun ne se cantonne à travailler sur son champ de connaissance préalablement acquis en bachelor. Avec le Jigsaw teaching, les étudiants du groupe sont obligés d'acquérir une certaine maitrise des différents modules disciplinaires et concepts qui leur sont a priori étrangers, afin de réussir le travail collectif que les enseignants leur demandent de réaliser. Les apports disciplinaires s'assemblent alors comme les pièces d'un puzzle, dans l'ordre où ils se présentent, la « composition finale » n'étant révélée par et pour le groupe d'étudiants qu'une fois cet assemblage effectué (dans leur rapport écrit, ensuite évalué).

Une autre particularité de l'atelier est que cette immersion est organisée sur un même terrain, dans le Val d'Hérens, plus spécifiquement à Evolène et le Haut Val de Ferpècle (canton du Valais - Suisse ; figure 2), et ce, depuis la création du MUSE il y a dix ans. Ce choix a été fait pour divers motifs :

- avoir un terrain à examiner où il y a un minimum d'impacts humains, le programme étant initialement à dominante naturaliste ;

- mais dont l'accès est facile ;

- et où l'on trouve des conditions logistiques adéquates pour abriter un grand nombre d'étudiants et d'encadrants durant une semaine.

Le nombre total d'étudiants et d'encadrants a varié au cours des années, mais il avoisine en moyenne 48 étudiants et 21 encadrants (figure 3). En 2017, avec un nombre record de 75 étudiants, 23 encadrants dont des assistants (en thèse) ont animé l'atelier de terrain (figure 4), et une douzaine d'intervenants ont effectué des cours théoriques. 
Figure 2. Le Val d'Hérens (canton du Valais, Suisse) et les secteurs d'études des différents modules de l'atelier « Environnement alpin et sociétés » du MUSE

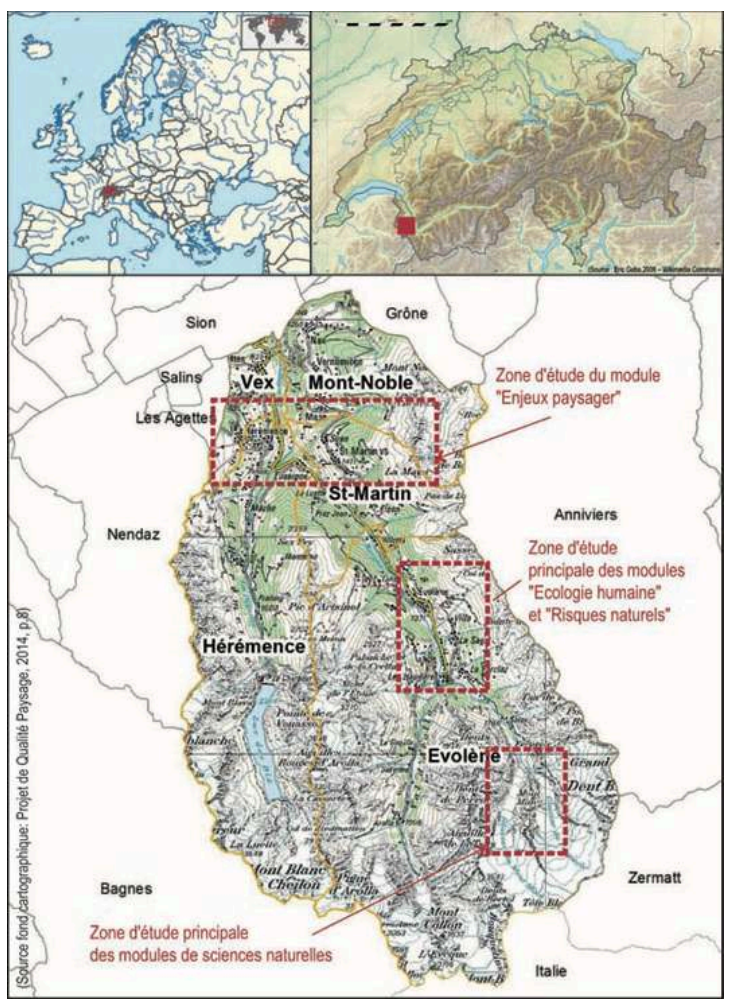

Figure 3. Évolution des effectifs d'étudiants, d'enseignants (y compris assistants), de leur ratio, et du nombre de modules enseignés dans l'atelier « Environnement alpin et sociétés » de 2007 à 2017

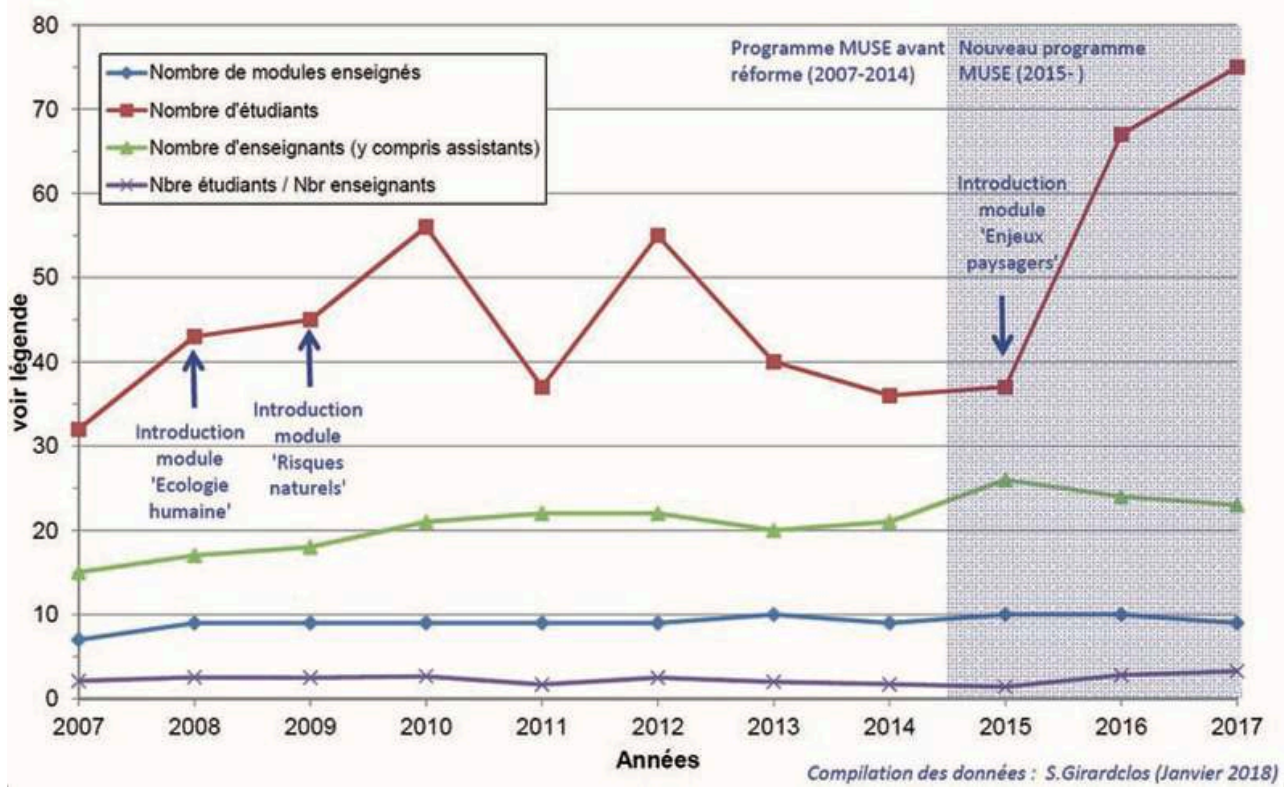


Figure 4. Étudiants et encadrants lors de l'atelier de terrain à Evolène en octobre 2017

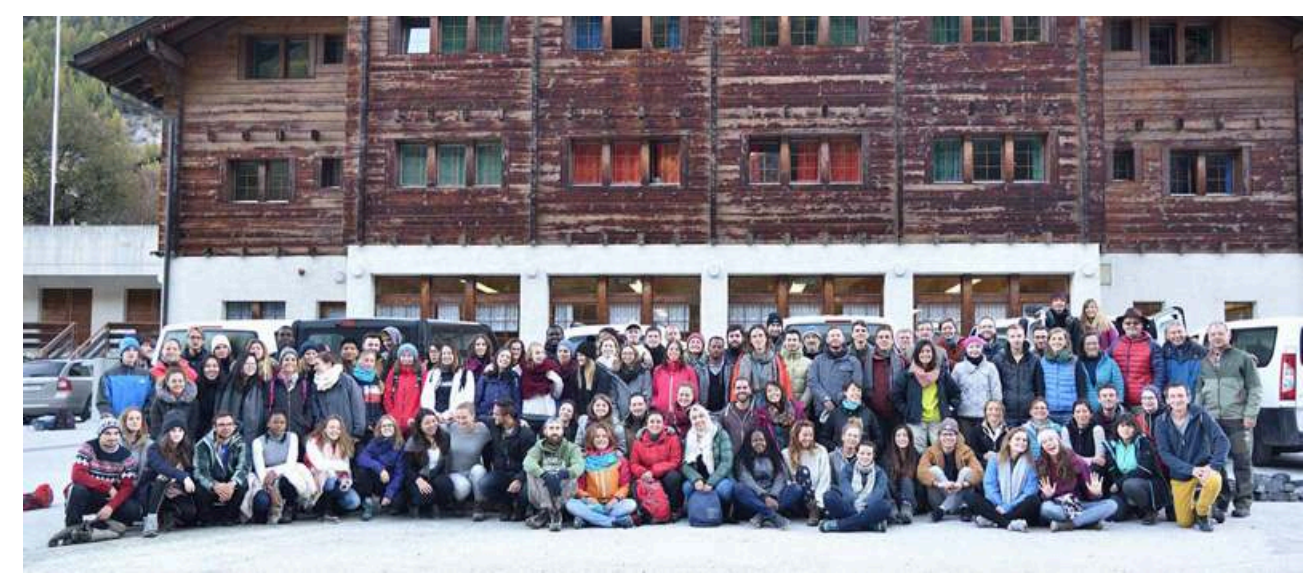

Photo : Jean-Luc Loizeau.

\section{Le choix du paysage, comme notion transversale}

11 Dès la deuxième année du MUSE, le choix du Val d'Hérens comme terrain d'enseignement s'est révélé pertinent au-delà du seul secteur du sandur ${ }^{5}$, des glaciers de Ferpècle et du mont Miné, démontrant aussi l'intérêt de la dimension socioculturelle et historique de la vallée avec l'ajout d'un module d'écologie humaine ${ }^{6}$. Puis, une réforme menée entre 2013 et 2015 pose le constat qu'il faut renforcer la contribution des sciences sociales dans la première année du programme du MUSE. À la faveur de diverses opportunités, en particulier le démarrage d'un projet de recherche intitulé "Didactique du paysage " au début 2015 dans le département de géographie et environnement ${ }^{7}$, il est décidé $d^{\prime y}$ introduire le paysage, notion perçue comme « intégrative » et «transversale » (Droz et al., 2009; Donadieu, 2007 et 2012). L'équipe de géographes choisis pour mener le «nouveau » module amène non seulement des compétences "paysage " mais permet également un apport de connaissances du terrain d'étude (voir notamment Petite, 2011). Un nouveau module «Enjeux paysagers » prend donc place dans le cours et dans l'atelier «Environnement alpin et sociétés » dès l'automne 2015.

\section{Le cadre imposé au module " enjeux paysagers »}

Le design du module " Enjeux paysagers » a été imposé par le programme d'étude et le format type des modules déjà existants, soit une trentaine d'heures de travail effectif, dont 8 heures de cours théoriques, une demi-journée de préparation au travail de terrain avec les étudiants " experts » de chaque groupe, un atelier sur le terrain avec les différents groupes (soit de 40 à 75 étudiants), et une demi-journée post-terrain avec les « experts » de chaque groupe d'étudiants pour préparer le rendu du rapport final. Les étudiants ont ensuite plusieurs jours de travail dédiés à la rédaction en groupe de leur rapport réunissant les différents modules thématiques à traiter.

13 Pour la partie atelier de terrain, un format d'enseignement d'une journée-bloc dévolue aux « Enjeux paysagers » est imposé par les contraintes logistiques des autres modules disciplinaires. En effet, les modules disciplinaires fonctionnent comme des entités indépendantes et sont répétés sur quatre jours successivement à l'intention de 
plusieurs groupes d'étudiants (figure 5). Ainsi, la journée «Enjeux paysagers » est symboliquement positionnée " en charnière ", au milieu de la semaine (le mercredi), et permet un échange entre tous les étudiants et tous les enseignants au sein d'un ou deux grand(s) groupe(s).

D'autres choix, plus contraints que voulus, ont été effectués en lien en particulier avec le doublement des effectifs initiaux de 30-35 vers 70-75 étudiants en 2016 et 2017. La partie théorique du cours «Enjeux paysagers » a ainsi été réduite au profit d'apports méthodologiques plus importants en amont, destinés à mieux préparer l'ensemble des étudiants à l'exercice d'analyse des paysages lors de l'atelier de terrain.

Figure 5. Planning de la semaine d'atelier de terrain, montrant l'alternance des modules pour chaque groupe d'étudiants (octobre 2017)

\begin{tabular}{|c|c|c|c|c|c|c|c|}
\hline Groupes & lundi 9 oct.17 & mardi 10 oct. 17 & mercredi 11 oct. 17 & \multicolumn{2}{|c|}{ jeudi 12 oct. 17} & \multicolumn{2}{|c|}{ vendredi 13 oct. 17} \\
\hline $1 a$ et $1 b$ & végétation alpine & hydrobiologie - algues & & $\begin{array}{l}\text { enquêtes } \\
\text { sociales }\end{array}$ & $\begin{array}{l}\text { risques } \\
\text { naturels }\end{array}$ & \multicolumn{2}{|c|}{$\begin{array}{l}\text { géologie glaciaire et } \\
\text { lichenométrie }\end{array}$} \\
\hline $2 a$ et $2 b$ & hydrobiologie - algues & végétation alpine & & $\begin{array}{l}\text { risques } \\
\text { naturels }\end{array}$ & $\begin{array}{l}\text { enquêtes } \\
\text { sociales }\end{array}$ & \multicolumn{2}{|c|}{$\begin{array}{l}\text { glaciologie et } \\
\text { météorologie }\end{array}$} \\
\hline $3 a$ et $3 b$ & $\begin{array}{l}\text { hydrobiologie - } \\
\text { invertébrés }\end{array}$ & $\begin{array}{l}\text { glaciologie et } \\
\text { météorologie }\end{array}$ & & \multicolumn{2}{|c|}{ végétation alpine } & $\begin{array}{l}\text { risques } \\
\text { naturels }\end{array}$ & $\begin{array}{l}\text { enquêtes } \\
\text { sociales }\end{array}$ \\
\hline $4 a$ et $4 b$ & $\begin{array}{l}\text { hydrologie et } \\
\text { sédimentologie }\end{array}$ & $\begin{array}{l}\text { hydrobiologie - } \\
\text { invertébrés }\end{array}$ & & \multicolumn{2}{|c|}{$\begin{array}{l}\text { glaciologie et } \\
\text { météorologie }\end{array}$} & $\begin{array}{l}\text { enquêtes } \\
\text { sociales }\end{array}$ & $\begin{array}{l}\text { risques } \\
\text { naturels }\end{array}$ \\
\hline $5 a$ et $5 b$ & $\begin{array}{l}\text { écologie humaine: } \\
\text { enquêtes sociales }\end{array}$ & $\begin{array}{c}\text { géologie glaciaire et } \\
\text { lichenométrie }\end{array}$ & & \multicolumn{2}{|c|}{$\begin{array}{l}\text { hydrobiologie - } \\
\text { invertébrés }\end{array}$} & \multicolumn{2}{|c|}{$\begin{array}{l}\text { hydrologie et } \\
\text { sédimentologie }\end{array}$} \\
\hline $6 a$ et $6 b$ & $\begin{array}{l}\text { écologie humaine: } \\
\text { enquêtes sociales }\end{array}$ & $\begin{array}{l}\text { hydrologie et } \\
\text { sédimentologie }\end{array}$ & $E$ & \multicolumn{2}{|c|}{ végétation alpine } & \multicolumn{2}{|c|}{$\begin{array}{l}\text { hydrobiologie - } \\
\text { invertébrés }\end{array}$} \\
\hline $7 a$ et $7 b$ & $\begin{array}{l}\text { glaciologie et } \\
\text { météorologie }\end{array}$ & $\begin{array}{l}\text { écologie humaine: } \\
\text { enquêtes sociales }\end{array}$ & & \multicolumn{2}{|c|}{$\begin{array}{l}\text { géologie glaciaire et } \\
\text { lichenométrie }\end{array}$} & \multicolumn{2}{|c|}{ végétation alpine } \\
\hline $8 a$ et $8 b$ & $\begin{array}{l}\begin{array}{c}\text { géologie glaciaire et } \\
\text { lichenométrie }\end{array} \\
\end{array}$ & $\begin{array}{l}\text { écologie humaine: } \\
\text { enquêtes sociales }\end{array}$ & & \multicolumn{2}{|c|}{$\begin{array}{l}\text { hydrologie et } \\
\text { sédimentologie }\end{array}$} & \multicolumn{2}{|c|}{ végétation alpine } \\
\hline $9 a$ et $9 b$ & $\begin{array}{c}\text { géologie glaciaire et } \\
\text { lichenométrie }\end{array}$ & végétation alpine & & \multicolumn{2}{|c|}{$\begin{array}{l}\text { écologie humaine: } \\
\text { enquêtes sociales }\end{array}$} & \multicolumn{2}{|c|}{ hydrobiologie - algues } \\
\hline $10 a$ & végétation alpine & $\begin{array}{c}\text { géologie glaciaire et } \\
\text { lichenométrie }\end{array}$ & & \multicolumn{2}{|c|}{ hydrobiologie - algues } & \multicolumn{2}{|c|}{$\begin{array}{l}\text { écologie humaine: } \\
\text { enquêtes sociales }\end{array}$} \\
\hline
\end{tabular}

\section{Un parti pris méthodologique affirmé pour l'atelier « Enjeux paysagers »}

15 Afin de considérer tant les aspects matériels qu'immatériels du système paysager, et en lien avec la Convention européenne du paysage (CEP) de 2000, les responsables du module «Enjeux paysagers " imaginent des modalités pédagogiques proposant une "sensibilisation au territoire et à l'environnement", pour mieux cerner les conséquences des actions et décisions en lien avec les politiques environnementales et de développement (Davasse, 2006). Plutôt que d'envisager la seule approche descriptive des paysages, le choix s'est en particulier dirigé vers une confrontation des regards des étudiants entre eux sur le terrain, mais aussi avec des témoignages d'acteurs locaux in situ, afin de cerner la pluralité des points de vue (Partoune, 2004) et de discuter des paysages en présence.

16 Un itinéraire sur le terrain est rapidement arrêté par les deux responsables « Enjeux paysagers ", sous la forme d'un transect en aval de la vallée, dans une zone habitée où s'illustrent en vis-à-vis des évolutions paysagères différenciées de deux communes ayant eu des stratégies de développement différentes. Ce contraste paraît propice à discuter des trajectoires suivies, des conséquences des choix de développement et des paysages résultant de ces choix, ainsi que des perspectives et impacts paysagers futurs. 
Lors de la journée dédiée à l'exercice d'analyse du paysage, des rencontres avec des acteurs locaux ont donc lieu sur chacun des deux versants, à savoir les communes d'Hérémence et de Saint-Martin. Des temps sont prévus également pour que les étudiants observent seuls et/ou en groupe le paysage, le dessinent pour mieux en cerner les composants, et interagissent avec les enseignants présents afin de chercher des facteurs explicatifs.

La méthode de travail imposée aux étudiants sur le terrain, par le croquis et le dessin, implique chacun d'entre eux dans le travail de son groupe et aide à une meilleure analyse du paysage. Cette dimension de l'intéressement, de l'implication de tous dans le rendu demandé (un bloc-diagramme imposé dans le rapport à produire, assorti d'une synthèse écrite devant présenter et situer les enjeux paysagers identifiés sur le transect), fait qu'au retour du terrain, les étudiants doivent réinvestir les éléments collectés pour en tirer des enseignements, et travailler une «représentation» modélisée à partir :

- des observations faites au sein de chaque groupe ;

- des dires d'acteurs rencontrés ;

- des données et sources d'information consultées (une sélection leur est proposée - voir ciaprès) ;

- voire d'éléments issus des autres modules disciplinaires (relevés de végétation par exemple).

Le cours et la préparation en amont du terrain vont viser à poser les bases théoriques et méthodologiques nécessaires pour effectuer l'analyse paysagère attendue par les différents groupes.

\section{Au contour et au détour d'une expérience singulière}

\section{Le paysage comme « mise en relation » entre disciplines}

Certes, la journée de terrain «Enjeux paysagers » a pu générer quelques frustrations chez les étudiants : les deux tiers ont notamment mentionné dans l'enquête menée en février 2017 qu'ils "manquaient de temps sur place», des "trajets longs et des retards » ou encore un "manque de ressources humaines pour encadrer chacun des groupes ». Néanmoins, il est à noter que la majorité des étudiants $(90 \%)$ a exprimé beaucoup d'intérêt suite à la découverte de la notion de paysage. Ils y ont principalement apprécié la discussion de «points de vue différents mais complémentaires » des acteurs sur site, le travail proposé visant à analyser et à comprendre différemment l'environnement alpin, et souligné l'intérêt du transect étudié, révélateur d'enjeux paysagers différenciés (figure 6). Au final, ils ont vécu une « expérience enrichissante » et appris à poser un regard à la fois différent et différencié sur cette vallée ( $c f$. enquête février 2017). Les échanges entre enseignants ont montré que cet enthousiasme est aussi présent chez les intervenants qui ont accompagné ce nouveau module, et dès la première sortie de terrain en 2015 , alors que la lecture du paysage s'est déroulée dans un épais brouillard! Cette situation météorologique a été pour tous un événement marquant, une expérience du lieu singulière, où la découverte du paysage a eu lieu au fil de cette fameuse journée, le paysage se dévoilant au sens propre et figuré, au gré des dires et des rencontres d'acteurs. 
Figure 6. Vue panoramique annotée (prise au-dessus d'Euseigne) du transect paysager entre les communes de Saint-Martin et d'Hérémence

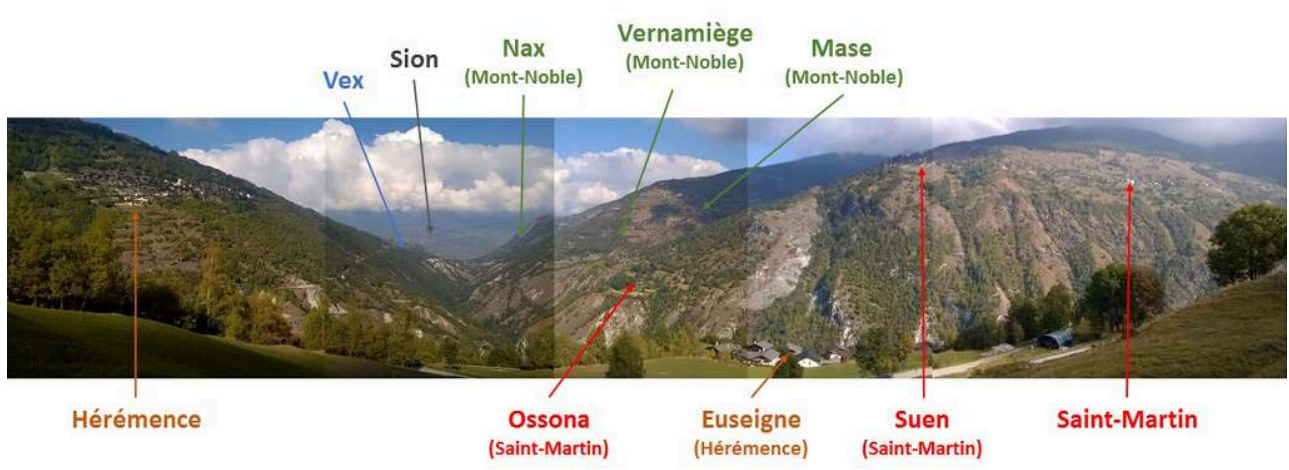

Photomontage : Sylvie Paradis, 2016).

La contrainte de la configuration du terrain (impactant les temps de parcours, etc.) rend l'exercice d'enseignement compliqué sur une journée, et ceci même en se concentrant sur une portion réduite de la vallée. La complexité du sujet et le temps limité de l'exercice ont obligé à envisager un travail plus conséquent de préparation en amont. Pour se faire, une sélection de ressources, nombreuses et riches (supports vidéos, extraits d'entretiens, données ciblées, articles scientifiques, etc.), a permis d'orienter et de nourrir en un temps réduit la réflexion des étudiants en leur permettant de s'initier à l'analyse des enjeux paysagers. En effet, la synergie avec le projet de recherche "Didactique du paysage" a permis de constituer une "boîte à outils " pertinente, avec des données, des extraits vidéos, des textes scientifiques ou issus de la presse locale, des photographies anciennes et récentes, etc., qui s'est révélée particulièrement efficace pour optimiser l'exercice pédagogique du module «Enjeux paysagers » et aider les étudiants sans les surcharger d'informations. Cette préparation en amont du terrain et en complément des observations menées in situ a été soulignée dans les évaluations menées auprès des étudiants comme très bénéfique pour mieux appréhender les différentes réalités du terrain (notamment l'évaluation ADEVEN de 2016).

Les attendus en termes d'analyse demeurent toutefois modestes vu le contexte (diversité des profils étudiants, nombre d'heures réduit, etc.), l'apprentissage de la lecture du paysage étant déjà une étape en soi. Les rapports corrigés, d'une année à l'autre, sont là, néanmoins, pour montrer que l'accompagnement pédagogique est plus performant dans ces choix chaque année, car les étudiants arrivent à mieux établir des liens entre les disciplines (dans la conclusion générale des rapports entre autres), voire développent leur analyse au-delà de ce qui est demandé, nous réservant de belles surprises en intégrant une dimension sensible plus poussée ${ }^{8}$.

Le transect paysager choisi se révèle également être un véritable "cas d'école ", donnant à voir et à discuter de l'état, des dynamiques comme des enjeux paysagers des deux versants en vis-à-vis. Mieux encore, les autres intervenants du MUSE participant à la journée du module "Enjeux paysagers ", en tant que chauffeurs des minibus, y découvrent des liens à faire avec leurs propres modules, allant dans le sens des attentes d'interdisciplinarité. Ainsi, en octobre 2017, des interventions spontanées ont lieu pour guider la lecture du paysage en croisant plusieurs entrées disciplinaires sur un même point de vue (notamment sur la géomorphologie glaciaire). D'autres ont été surpris de découvrir, sur ce secteur géographique habité, des liens avec leurs enseignements qu'ils 
ne pouvaient pas faire au pied des glaciers (figure 7). Sans avoir expérimenté le transect paysager, ces liens entre modules disciplinaires n'auraient pas été aussi clairement identifiés, ce qui démontre l'importance d'un dialogue entre disciplines in situ, ni seulement destinés aux seuls étudiants, mais aux enseignants également. Il s'agit là de la révélation de toute la force et de la pertinence du travail de terrain, qui permet - au sens propre comme au figuré - toutes les rencontres. C'est en ce sens que la notion de paysage, avec ce module «Enjeux paysagers ", est devenue une sorte de laboratoire, au sens d'une expérimentation itérative sur le terrain et sur trois années consécutives, pour mieux fédérer les disciplines entre elles.

Figure 7. Profil schématique longitudinal du Val d'Hérens, avec indication des secteurs d'études des différents modules de l'atelier « Environnement alpin et sociétés »

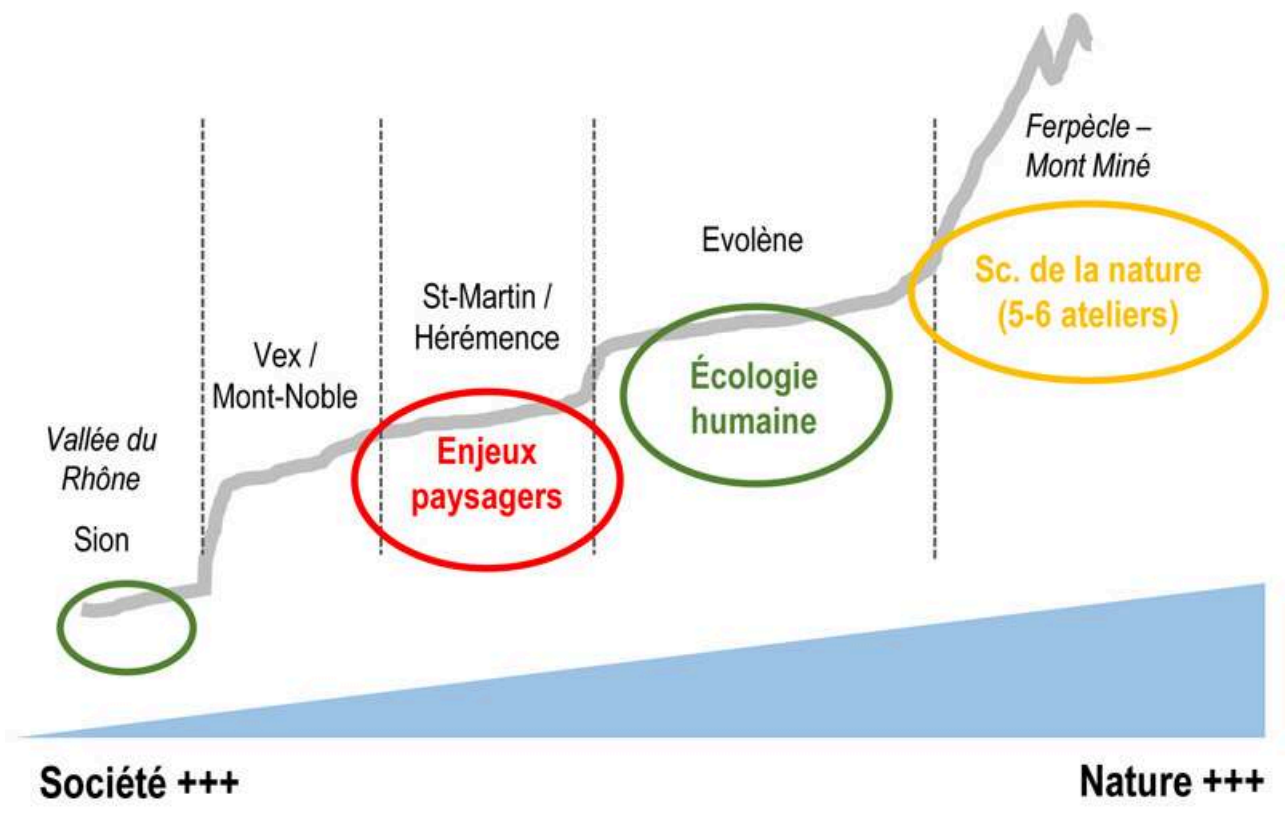

Source : Sylvie Paradis, 2017

Cette rencontre a également eu lieu lors de l'atelier d'octobre 2017, quand l'intervenante paysage s'est rendue en observation sur les terrains d'autres modules thématiques (risques naturels, écologie humaine, lichenométrie et géologie glaciaire, climatologie et glaciologie). Ces échanges pédagogiques en sens inverse ont permis d'envisager de nouveaux liens avec le module « Enjeux paysagers ». En voyant les outils manipulés (dont des GPS), le déroulé pédagogique, le type de mesures effectuées ou les tableaux de saisie de données utilisés dans les différents modules (figure 8), des pistes d'amélioration ont pu être identifiées en termes d'instrumentation de la production de l'analyse du paysage, qui pourraient être plus « en phase » avec les modules en sciences naturelles, traditionnellement très normés et instrumentés. Il est par exemple envisagé d'utiliser, dans le futur, les marques annuelles du retrait glaciaire, peintes sur des blocs laissés par le glacier ou des roches moutonnées lors des précédents ateliers, pour faire prendre conscience des impacts sur le terrain du changement climatique (figure 9). L'expérience «du terrain des autres» devient alors essentielle pour mieux projeter l'exercice pédagogique dans ses dimensions interdisciplinaires. Toutefois, il faut noter que cette ouverture demande du temps, une volonté et un état d'esprit ouvert, afin que 
la rencontre et un enrichissement mutuel soient possibles. Elle a donc comme base et prérequis la volonté commune de vouloir expérimenter l'enseignement collectivement.

Pour aller plus loin dans cette recherche d'interdisciplinarité, à l'automne 2017, est discutée la mise en place d'un cahier (ou carnet) de terrain commun à tous les modules, outil de «transversalité » issu du module «Enjeux paysagers » pour consigner toutes les données et observations chemin faisant.

Figure 8. Des modalités et outillages différents entre modules de sciences naturelles et de sciences sociales
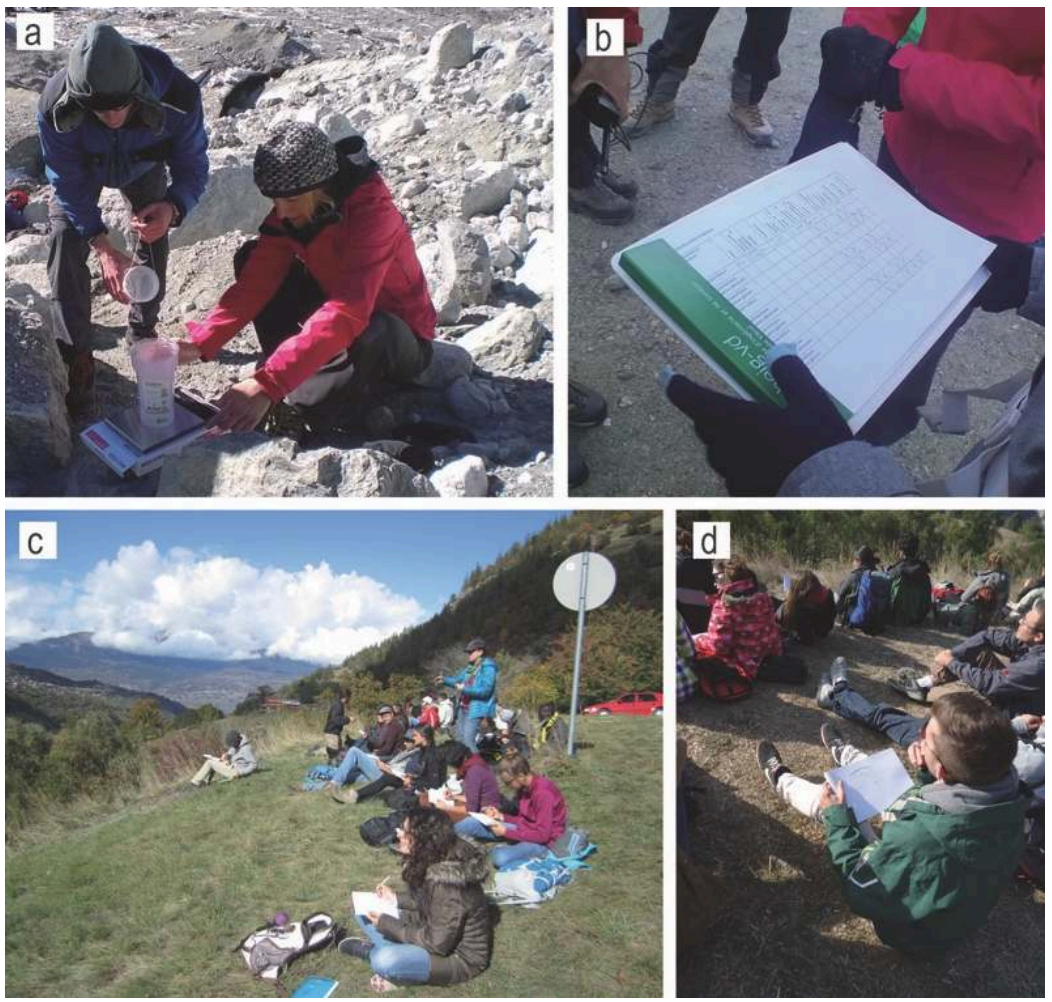

Exemple du module «Glaciologie et météorologie » : a. balance et b. tableau de saisie de données ; exemple du module « Enjeux paysagers » : c. lecture de paysage in situ et d. croquis et carnet de terrain.

8a et 8b : photos Sylvie Paradis, 2017 ; 8c : photo David McCrae, 2015 ; 8d : photo Sylvie Paradis, 2016. 
Figure 9. Traces de peinture réalisées annuellement pour marquer le recul du glacier du Mont-Miné
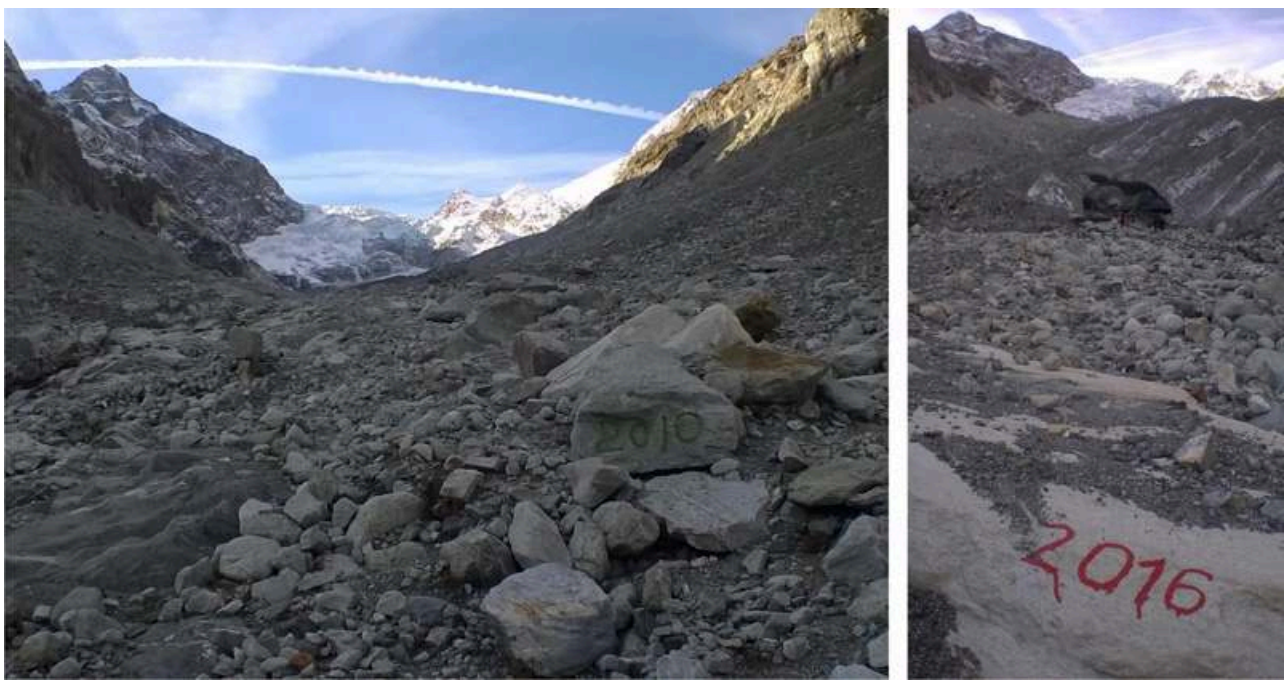

Photos : Sylvie Paradis, 2017.

\section{Un changement d'échelle : du micropaysage au grand paysage...}

Une autre dimension pertinente de l'analyse du paysage apparue avec l'enseignement de l'atelier « Environnement alpin et sociétés » est celle des différences d'échelles. Par rapport à tous les autres modules de l'atelier, le module « Enjeux paysagers » a ouvert un cadre d'observation plus large sur l'ensemble de la vallée. À cela s'ajoute la complexité liée à la notion de paysage, qui ouvre à une multitude d'angles d'analyse, telles les diverses opportunités décisionnelles liées aux mouvements démographiques (vieillissement, exode rural, etc.), économiques (politique touristique, déprise agricole, etc.) ou environnementaux (protection de biotopes, risques naturels, etc.). Se retrouver face à un paysage peut ainsi déstabiliser, les étudiants ne sachant plus ou pas par où commencer leur observation ni comment ils vont cerner les enjeux paysagers. Il faut les rassurer alors sur leurs capacités à pouvoir le faire, les accompagner selon les questions qu'ils se posent in situ.

Un autre des principaux apports $d u$ paysage dans le contexte $d u$ cours «Environnement alpin et sociétés », et plus largement dans le programme du MUSE, a peut-être été de permettre de contextualiser, de visualiser et de faire prendre conscience des dynamiques (naturelles et sociales) à l'œuvre dans l'observation et l'évocation du changement climatique. Mais ce point reste à optimiser dans les années à venir. Le module «Enjeux paysagers » a néanmoins permis aux étudiants de mieux cerner, sur le terrain, les conséquences des actions et des décisions en lien avec les politiques environnementales et de développement territorial (Davasse, 2006) dans le Val d'Hérens, comme en témoignent notamment les rapports rendus.

\section{Conclusion}

Un temps d'acculturation est nécessaire pour optimiser toute expérience pédagogique, cela est encore plus vrai pour un programme visant à développer l'interdisciplinarité comme celui du MUSE avec ses modalités spécifiques tel le Jigsaw teaching. En ce qui 
concerne le module « Enjeux paysagers » du cours et de l'atelier « Environnement alpin et sociétés ", la démarche pédagogique a pu être accélérée et améliorée grâce à l'exercice réflexif et collaboratif mené par les auteurs, dans l'idée d'un enrichissement mutuel et d'une exploration par le paysage pour créer plus de ponts entre disciplines sur le terrain.

Par ailleurs, le projet de recherche concomitant sur la «Didactique du paysage » a constitué une véritable opportunité, tant pour l'enseignement interdisciplinaire du paysage que pour le projet de recherche lui-même, amenant une posture d'observation, d'échanges sur les pratiques et une réflexivité que l'on se permet rarement en tant qu'enseignant dans le «feu de l'action». Cela a aussi participé de l'idée du paysage notion-laboratoire. La «symbiose » entre recherche et formation prend tout son sens ici, en particulier l'enrichissement tant de la recherche et de l'enseignement rendu possible par les allers et retours constants entre les deux. Néanmoins, cela n'aurait pas eu le même impact sans la rencontre entre enseignants d'horizons disciplinaires variés et l'envie partagée d'aller vers plus d'interdisciplinarité et un esprit d'ouverture aux méthodes des autres.

L'introduction d'un nouveau module «Enjeux paysagers » en 2015 a ainsi ouvert des perspectives nouvelles dans l'atelier et le cours "Environnement alpin et sociétés ", en particulier par le changement d'échelle opéré et le rapport au terrain établi. Le paysage a ainsi permis de démontrer que l'environnement est aussi une construction sociale, avec différentes facettes, en questionnant les regards portés, que ce soit ceux des acteurs-témoins rencontrés, des intervenants ou des étudiants eux-mêmes, ou les différentes entrées disciplinaires, pour identifier ce qui peut faire sens.

Ce choix du paysage, à l'interface « entre nature et société » (Brunet, 1974; Bertrand, 1978), est véritablement apparu à travers cette expérience dans le MUSE comme une opportunité d'explorer "une expression de la construction politique, sociale et culturelle des rapports humains à l'espace » (Donadieu, 2007) et pour réinterroger la nature et l'environnement en lien avec les activités humaines (Bertrand et Bertrand, 2014). Il n'en demeure pas moins qu'il reste encore beaucoup à faire pour tisser des liens entre les modules enseignés dans le cours et l'atelier "Environnement alpin et sociétés ", y compris entre ceux des sciences sociales. Cette rencontre et cette prise de conscience sont déjà de grands pas vers un enseignement plus interdisciplinaire.

\section{BIBLIOGRAPHIE}

Aronson, E., The Jigsaw strategy, San Diego, Academic Press, 2002.

Aronson, E., The Jigsaw Classroom, Thousand Oaks, Sage Publications/University of Michigan, 1978.

Bertrand, C., Bertrand, G., « La nature-artefact : entre anthropisation et artialisation, l'expérience du système GTP (Géosystème-Territoire-Paysage) ", L'Information géographique, n 3, vol. 78, 2014, p. 10-25.

Bertrand, G., « Le paysage entre la Nature et la Société », RGPSO, vol. 49, n² 2, 1978, p. 239-258. 
Brunet, R., « Analyse des paysages et sémiologie. Éléments pour un débat », L’Espace géographique, vol. 3, n² 2, 1974, p. 120-126.

Clarke, J., Pieces of the puzzle: the Jigsaw teaching, Westport, Greenwood Press, 1994.

Davasse, B., « La notion de paysage, éléments de réflexion pour une pédagogie dans le domaine du paysage ", dans Bichindariz, F., Qu'est-ce que le paysage ?, vol. 2, Rennes, 2006, p. 38-42.

Donadieu, P., Sciences du paysage, entre théories et pratiques, Cachan, Lavoisier, coll. « Tec et Doc », 2012.

Donadieu, P., « Le paysage, les paysagistes et le développement durable : quelles perspectives ?», Économie rurale, $\mathrm{n}^{\circ}$ 297-298, janvier-mars 2007, p. 10-22.

Droz, Y., Mieville-Ott, V., Forney, J., Spichiger, R., Anthropologie politique du paysage. Valeurs et postures paysagères des montagnes suisses, Paris, Karthalia, coll. « Tropiques », 2009.

Efrancey Dao, N., Wildi, W., « Rapport d'auto-évaluation du programme Maîtrise universitaire en sciences de l'environnement (MUSE) ", université de Genève, comité d'auto-évaluation MUSE, 2013.

ISE - Institut des sciences de l'environnement de l'université de Genève, «Syllabus MUSE. Année 2016-2017».

Lawrence, R., « Human Ecology », dans Tolba, M. K. (ed.), Our Fragile World: Challenges and Opportunities for Sustainable Development, vol. 1, Oxford, EOLSS Publishers, 2001, p. 675-693.

Partoune, C., «L'approche du paysage revisitée à la lumière des théories sur les styles d'apprentissage », « Journées nationales d'études didactiques de l'histoire de la géographie », Caen, 2004, p. 123-150.

Petite, M., Identités en chantiers dans les Alpes. Des projets qui mobilisent objets, territoires et réseaux, Berne, Ed. Peter Lang, 2011.

Rege Collet, N., « Enseignement interdisciplinaire : le défi de la cohérence pédagogique », « $20^{\mathrm{e}}$ Congrès international de l'AIPU. L'université au service de l'apprentissage : à quelles conditions?", 2003, p. 1-23.

Resweber, J.-P., « Les enjeux de l'interdisciplinarité », Questions de communication, nº 19, 2011, p. 171-200.

Reverdy, C., «Éduquer au-delà des frontières disciplinaires ", Dossier de veille de l'IFÉ, n 100 , mars 2015.

Sainte Fleur, K., Mencacci, N., Castera, J., « Effets de la méthode coopérative par la technique jigsaw dans l'enseignement/apprentissage actuel », Educational Journal of the University of Patras, Unesco Chair, vol. 3, n 2, 2016, p. 61-68.

\section{NOTES}

1. ECTS : European Credit Transfer Scale.

2. ADEVEN est le service de l'université de Genève qui permet une évaluation des enseignements par les étudiants. Cette évaluation se fait par questionnaire et est anonyme.

3. Voir les présentations en ligne du MUSE: http://masters.unige.ch/mastersinterdisciplinaires\#sciences-de-l-environnement-muse et https://www.unige.ch/muse/. 
4. En 2015 (37 élèves), il y aura 10 groupes de 3-4 étudiants. En 2016 (67 élèves), ce seront 10 groupes de 6-7 étudiants, subdivisés en 10 sous-groupes de 3-4. Puis en 2017 (75 élèves), 10 groupes de 7-8 étudiants, subdivisés en 19 sous-groupes de 3-4.

5. Plaine d'épandage constituée d'alluvions glaciaires déposées par les eaux s'échappant d'un glacier.

6. L'écologie humaine fait référence à l'étude des interdépendances dynamiques entre les populations humaines et les éléments physiques, biotiques, sociaux et culturels de leur environnement et de la biosphère (Lawrence, 2001). Auparavant ce module de sciences sociales s'intitulait: «enquêtes sociales".

7. Il s'agit du projet FNS (Fonds national suisse) «Didactique du paysage. Mutualisation des expériences et perspectives didactiques à propos des controverses paysagères ». Ce projet de recherche va s'intéresser entre autres (ou notamment) à la controverse qui a eu lieu autour de l'échec d'un projet de Parc naturel régional (PNR) dans le Val d'Hérens après votation en 2011.

8. À l'automne 2017, un groupe d'étudiants a volontairement développé une approche sensible olfactive du paysage en plus du travail demandé, en s'appuyant sur les compétences en neurosciences d'un des membres du groupe. Les approches sensibles sont évoquées en cours mais non imposées dans l'exercice de terrain.

\section{RÉSUMÉS}

Depuis l'automne 2015, un module « Enjeux paysagers » a été introduit au premier semestre du master universitaire en sciences de l'environnement (MUSE), formation interdisciplinaire et interfacultaire de l'université de Genève (Suisse). Ce nouveau module intervient au cours d'une première étape d'initiation à l'interdisciplinarité et d'immersion aux thématiques de l'environnement. Ce texte revient sur cette expérience pédagogique, dans un esprit réflexif partagé par les auteurs, tous intervenants dans le MUSE, et issus de différentes disciplines. Le paysage est placé ici comme «notion-laboratoire » pour favoriser les ponts entre les disciplines enseignées et améliorer la mise en avant d'une interdisciplinarité, voire d'une transdisciplinarité, entre sciences de la société et sciences naturelles et exactes.

In the autumn of 2015, a module entitled "Landscape Issues" (in French, "Enjeux paysagers") was introduced in the first semester of the University Master of Environmental Sciences (in French, Master Universitaire en Sciences de l'Environnement, MUSE), an interdisciplinary and interfaculty training course at Geneva University (Switzerland). This new module is part of a first stage in the initiation to the interdisciplinary study of environmental themes. The article describes this teaching experiment in a reflective mode shared by the authors, all of whom are teachers of different disciplines and work in the MUSE programme. The landscape is positioned here as a "laboratory-concept" for fostering links between the disciplines taught and promoting interdisciplinarity, or transdisciplinarity, between social, natural and exact sciences.

\section{INDEX}

Mots-clés : analyse paysagère, master universitaire, interdisciplinarité, atelier de terrain

Keywords : landscape analysis, university master, interdisciplinarity, field workshop 


\section{AUTEURS}

\section{SYLVIE PARADIS}

Sylvie Paradis, géographe et architecte-urbaniste, est collaboratrice scientifique au département de géographie et environnement de la faculté des sciences de la société - université de Genève, et chercheure associée dans l'UMR Territoires de Clermont-Ferrand (issue du rapprochement des UMR Métafort et du Céramac).

sylvie.paradis[at]unige[dot]ch et sylvie.paradis[at]wanadoo[dot]fr

\section{STÉPHANIE GIRARDCLOS}

Stéphanie Girardclos est docteure en sciences de la terre, géologue du quaternaire et limnogéologue. Elle est maître d'enseignement et de recherche au département des sciences de la terre et à l'Institut des sciences de l'environnement (ISE) de l'université de Genève.

stephanie.girardclos[at]unige[dot]ch

\section{CÉDRIC LAMBERT}

Cédric Lambert, sociologue, est chargé d'enseignement à l'Institut des sciences de l'environnement (ISE) de l'université de Genève.

cedric.lambert[at]unige[dot]ch

\section{MATHIEU PETITE}

Mathieu Petite, docteur en sciences économiques et sociales, est géographe et chargé de cours au département de géographie et environnement, université de Genève.

mathieu.petite[at]unige[dot]ch

\section{MARJORIE PERROUD}

Marjorie Perroud est docteure en sciences de l'environnement, chercheuse en climatologie, et collaboratrice scientifique à l'Institut des sciences de l'environnement (ISE) de l'université de Genève.

marjorie.perroud[at]unige[dot]ch 